\title{
Zur Debatte um ungegenständliche Kunst in den 50er Jahren: Das »Leverkusener Gespräch»
}

\author{
Christoph Zuschlag
}

»Vermutlich hat noch nie am runden Tisch eines Funkhauses hierzulande eine so illustre Versammlung von Kunstkennern gesessen wie heute hier in Köln. "Mit diesen Worten eröffnete Rolf Wiesselmann am 1. Dezember 1956 ein Rundfunkgespräch - und er übertrieb beileibe nicht! Denn es waren tatsächlich einige der renommiertesten Kunstexperten Europas - aus Belgien, der Bundesrepublik, England, Frankreich, Italien, Holland und der Schweiz -, die in Köln zusammengekommen waren, um ein Gespräch fortzusetzen, das selbigen Tages in Leverkusen begonnen hatte: die Kunsthistoriker und Kunstkritiker Ernest Goldschmidt (Brüssel), Will Grohmann (Berlin), Giuseppe Marchiori (Venedig), Herbert Read (London), Michel Tapié (Paris) und Herta Wescher (Paris), sodann die Museumsleiter Pierre Janlet (Brüssel), Willem J. H. B. Sandberg (Amsterdam) und Georg Schmidt (Basel) und schließlich der junge Galerist Rodolphe Stadler (Paris). Außerdem nahm der Vorsitzende des Westdeutschen Künstlerbundes, Wilhelm Wessel, an der Runde teil. Worum ging es in dieser »illustren Versammlung «? Wodurch war sie zustande gekommen? Und wie läßt sich das Gespräch innerhalb der kunsttheoretischen Diskurse der 50er Jahre verorten? Dies sind die Leitfragen des vorliegenden Beitrages, der Licht auf ein spannendes, von der Forschung bislang vernachlässigtes Kapitel der deutschen Kunstgeschichte der 50er Jahre wirft. ${ }^{1}$

\section{Die Ausstellung "Malerei und Plastik in Westdeutschland - 1956 in Leverkusen}

Vom 28. November 1956 bis 2. Januar 1957 fand im Museum Morsbroich der Stadt Leverkusen die Ausstellung Malerei und Plastik in Westdeutschland - 1956 statt (Abb. 1). Veranstalter war der Westdeutsche Künstlerbund, der mit dieser Ausstellung sein zehnjähriges Bestehen feierte. Das Unternehmen war hochambitioniert, ging es

1 Vgl. zum folgenden auch Christoph Zuschlag, Wilhelm Wessel und Rodolphe Stadler - Ein deutsch-französischer Brückenschlag in den 1950er und frühen 1960er Jahren, in: Martin Schieder/Isabel Ewig (Hrsg.), KunstTransfer. Positionen zur deutsch-französischen Kunstgeschichte nach 1945, Berlin 2006 (im Druck). 


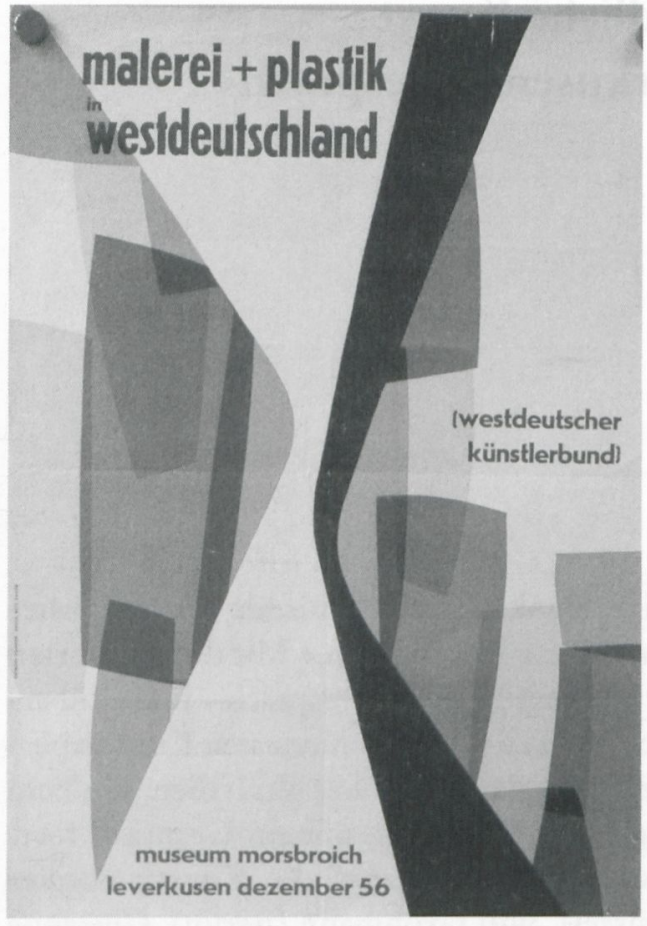

1 Ausstellungsplakat Malerei und Plastik in Westdeutschland - 1956, Museum Morsbroich, Leverkusen 1956/57

doch um eine Bestandsaufnahme der aktuellen Kunst in der Bundesrepublik. Verantwortlich für die Ausstellung, die unter der Schirmherrschaft des Ministerpräsidenten von Nordrhein-Westfalen, Fritz Steinhoff, stand und deren Kosten in Höhe von DM 12.000,- sich die Landesregierung Nordrhein-Westfalen und die Stadt Leverkusen teilten, war der Vorsitzende des Westdeutschen Künstlerbundes, der Iserlohner Maler Wilhelm Wessel. Museumsdirektor Curt Schweicher war für die Hängung der Ausstellung zuständig. Außerdem war er ebenso wie Wessel Mitglied einer achtköpfigen Jury, die über 400 Einsendungen von 80 Künstlerinnen und Künstlern zu beurteilen hatte. Weitere Juroren waren die Maler Karl Barth und Emil Schumacher, der Bildhauer Norbert Kricke und die Kunstkritiker bzw. -historiker Erhard Göpel, Gert Schiff und Albert Schulze Vellinghausen, wobei Barth, Kricke, Schumacher und Wessel Mitglieder des Westdeutschen Künstlerbundes waren. ${ }^{2}$

2 Die tatsächliche Zusammensetzung der Jury unterschied sich von der ursprünglich geplanten. So werden in einer Meldung der "Frankfurter Allgemeinen " vom 28. Juli 1956 auch der Bildhauer Karl Hartung und der Maler Fritz Winter als Mitglieder der Jury aufgeführt, die jedoch eine Mitarbeit offenbar ablehnten. Ein Foto der Jury bei der Arbeit veröffentliche die »Kölnische Rundschau « am 1. November 1956. Erfahrungsberichte über ihre Arbeit als Juroren publizierten Erhard Göpel in der Zeitung "Die Welt« vom 7. Dezember 1956 und Lambert Einhaus (wohl ein Pseudonym für Albert Schulze Vellinghausen) in der »Frankfurter Allgemeinen " vom 29. Dezember 1956. 
Der in einer Auflage von 600 Exemplaren erschienene Ausstellungskatalog verzeichnet 105 Exponate von 46 Künstlern und fünf Künstlerinnen (Sigrid Kopfermann, Brigitte Meier-Denninghoff, Emmy Roeder, Marie-Louise von Rogister, Irmgart WesselZumloh). ${ }^{3}$ Den Vorgaben entsprechend sollten sämtliche Exponate zwischen 1954 und 1956 in der Bundesrepublik entstanden sein, und in den wenigen Fällen, in denen der Katalog Datierungen verzeichnet, trifft dies auch zu. Wilhelm Wessel schreibt in seinem Katalogvorwort, die Ausstellung wolle »Tendenzen der westdeutschen Kunst von heute sichtbar« machen, wobei eine »jüngere Schicht« von Künstlern im Vordergrund stehe. Diese Einschätzung bestätigt sich beim Blick auf die Geburtsjahrgänge: Von den 51 teilnehmenden Künstlerinnen und Künstlern waren 16 nach 1920 geboren, 26 zwischen 1900 und 1920 und neun vor 1900. H. Schubert urteilte im »Kölner Stadtanzeiger« vom 6. Dezember 1956: »Die jüngeren Künstler - das sind vor allem die heute Dreißig- bis Fünfzigjährigen - aber sind in einer Art und Weise vertreten, wie sie keiner Künstlervereinigung im letzten Jahr gelang: Die Ausstellung [...] wurde zur gültigsten Dokumentation dessen, was >Moderne Kunst 1956 beinhaltet. « Dennoch, so Schubert weiter, gebe die Ausstellung kein "umfassendes Bild der Gesamtlage und sei »darum als Ausgangspunkt für eine grundlegende Beurteilung der gegenwärtigen Situation nur mit Einschränkungen geeignet «. Ungegenständlich arbeitende Künstler dominierten, wenngleich mit Erich Heckel auch ein Repräsentant des Brücke-Expressionismus vertreten war. $\mathrm{Zu}$ den figurativen Künstlern zählten weiterhin die Bildhauer Emmy Roeder, Gerhard Marcks, Fritz König und Hans Wimmer sowie HAP Grieshaber.

Die Ausstellung stieß in Presse und Öffentlichkeit sowohl auf Zustimmung als auch auf teilweise heftige Kritik. Dem hohen Anspruch, einen Überblick über die aktuelle Kunstszene zu bieten, widersprach nämlich der Umstand, daß viele bedeutende Künstler fehlten; zum einen ältere, bereits arrivierte Künstler wie Carl Buchheister, Werner Gilles, Georg Meistermann, Fritz Winter, Ernst Wilhelm Nay, Theodor Werner, Georg Mataré, Bernhard Heiliger, Karl Hartung und Hans Uhlmann, zum anderen Vertreter der jungen informellen Kunst wie Otto Greis, Heinz Kreutz, Peter Brüning, Winfred Gaul, Hann Trier, Gerhard Hoehme und Otto Herbert Hajek. Diese Künstler waren entweder nicht zur Teilnahme eingeladen oder ausjuriert worden, oder aber sie hatten die Ausstellung boykottiert - aber warum ein Boykott? Aus den im Stadtarchiv Leverkusen überlieferten Ausstellungsakten geht hervor, daß dies mit der Person Wessels und mit seinem eigenmächtigen Vorgehen bei der Ausstellungsvorbereitung zusammenhing. Nur auf persönliche Einladung konnten Künstler Werke einschicken, und wer eine solche Einladung erhielt, entschied allein Wessel. Da noch nicht einmal alle Mitglieder

3 Vgl. Malerei und Plastik in Westdeutschland - 1956. Ausstellung des Westdeutschen Künstlerbundes im Museum Schloß Morsbroich der Stadt Leverkusen, Bonn [1956]. Ein Exemplar des nicht paginierten Katalogs befindet sich im Stadtarchiv Leverkusen (StadtALev), wo ich Gabriele John für freundliche Unterstützung danke, unter Nr. LM 62. Darüber hinaus sind in Leverkusen zur Ausstellung folgende Akten vorhanden: StadtALev 410.311, StadtALev 410.312. StadtALev 5143.41-62 enthält ferner 17 Zeitungsartikel zu Ausstellung und Kritikertreffen. Sämtliche in diesem Aufsatz zitierten Presseartikel befinden sich in dieser Sammlung. 
des Westdeutschen Künstlerbundes eingeladen wurden, der ja Veranstalter der Ausstellung war, kam es zu Protesten innerhalb der Mitgliederschaft. Auch von den Nichtmitgliedern wollten sich einige wegen der dominanten Rolle Wessels nicht beteiligen, darunter die hoch angesehenen Künstler Werner Gilles und Karl Hartung, der damals Vorsitzender des Deutschen Künstlerbundes war. Dabei spielten offenbar auch die Erfahrungen eine Rolle, welche die Künstler mit Wessel im Jahr zuvor, 1955, in Paris gemacht hatten. Wessel hatte dort in Zusammenarbeit mit dem Galeristen René Drouin die Ausstellung Peintures et sculptures non figuratives en Allemagne d'anjourd'bui organisiert, die erste Übersichtsausstellung deutscher abstrakter Kunst im Ausland nach dem Krieg, und durch sein autokratisches Handeln so manchen Künstler gegen sich aufgebracht. ${ }^{4}$ Generelle Vorbehalte gab es gegen Wessel auch aufgrund seiner Vergangenheit als Parteimitglied der NSDAP und als Kriegsmaler. So stand die Leverkusener Ausstellung mehr als einmal auf der Kippe, und es war Wessels diplomatischem Geschick zu verdanken, daß sie gegen alle Widrigkeiten letztlich doch zustande kam. Allerdings trat Wessel 1957 wegen der Querelen um die Ausstellung vom Vorsitz des Westdeutschen Künstlerbundes zurück. ${ }^{5}$

\section{Das Expertengespräch in Leverkusen und Köln}

Von Anfang an hatte Wessel geplant, ausländische Experten um eine schriftliche Begutachtung der Ausstellung zu bitten und diese Gutachten zusammen mit den Pressekritiken zu veröffentlichen. So heißt es in dem Einladungsschreiben an die Künstler: "Die Zusammenfassung dient der Information des In- und Auslandes und dem Zweck weiterer Belebung des internationalen Kunstausschusses sowie der steigenden Ausstellungsmöglichkeit deutscher Künstler im Ausland. $\aleph^{6}$ Und in seinem oben zitierten Katalogvorwort schreibt Wessel: »Heute besteht für uns nicht mehr die Isolierung wie seit 1933 oder auch kurz nach 1945. Ob wir gegenständlichen und ungegenständlichen Maler und Bildhauer im Gesamtbild der internationalen Kunstentwicklung noch eigene, nationale Züge aufweisen, mögen erfahrene, in- und ausländische Kenner besser beurteilen als wir. Wir werden den Kennern der internationalen aktuellen Kunst, die unsere Ausstellung besuchen, für ein unabhängiges, offenes Urteil gerade über diese Frage dankbar sein. « Die schriftlichen Stellungnahmen der Experten sowie die ausländische und inländische Presse zur Ausstellung wurden 1957 vom Westdeutschen Künstlerbund in einer eigenen, mit einer Umschlagzeichnung von Emil Schumacher versehenen Publi-

4 Vgl. Martin Schieder, René Drouin und seine Ausstellung »Peintures et sculptures non figuratives en Allemagne d'aujourd'hui 1955 in Paris, in: Uwe Fleckner/Martin Schieder/Michael F. Zimmermann (Hrsg.), Jenseits der Grenzen. Französische und deutsche Kunst vom Ancien Régime bis zur Gegenwart. Thomas W. Gaehtgens zum 60. Geburtstag, Band 3: Dialog der Avantgarden, Köln 2000, 180-200.

5 Vgl. Annette Müller-Held, Wilhelm Wessel 1904-1971, in: Erich Franz (Hrsg.), Wilhelm Wessel 1904-1971. Malerei - Materie, Köln 2002, 18-41, hier 38.

6 StadtALev 410.311, Teil 1, Blatt 9, verso. 
2 Umschlag der »Dokumentation

Westdeutscher Künstlerbund« von 1957 mit einer Zeichnung von Emil Schumacher

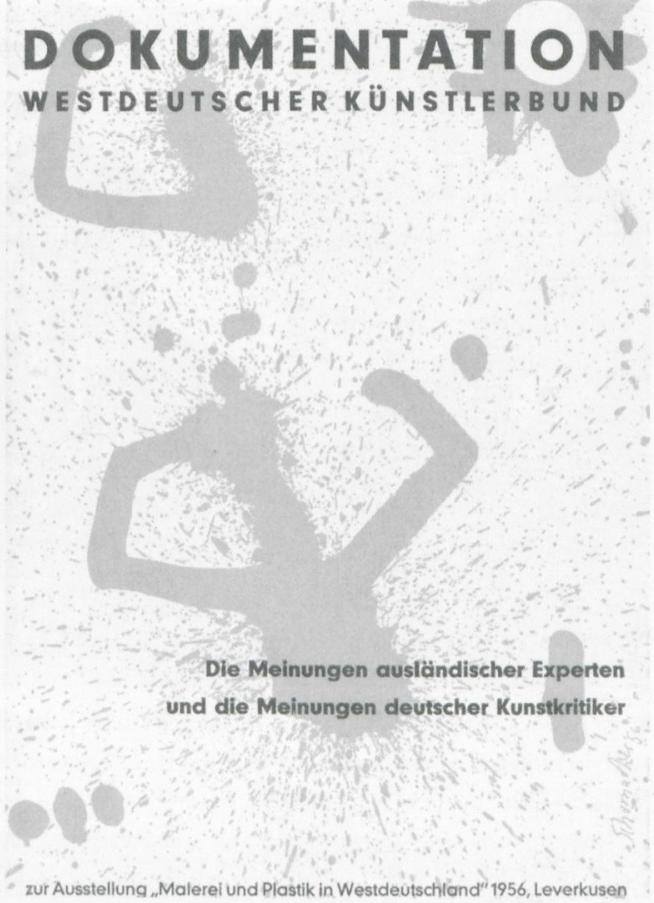

kation mit dem Titel »Dokumentation Westdeutscher Künstlerbund - Die Meinungen ausländischer Experten und die Meinungen deutscher Kunstkritiker - zur Ausstellung, Malerei und Plastik in Westdeutschland 1956, Leverkusen « dokumentiert (Abb. 2).?

Bereits drei Tage nach Eröffnung der Ausstellung, am Samstag, den 1. Dezember 1956, trafen auf Einladung Wilhelm Wessels im Museum Schloß Morsbroich die eingangs genannten zehn Kunstexperten zusammen (Abb. 3-5). An dem Treffen nahmen auch Wessel selbst, Vertreter des Kultusministeriums des Landes Nordrhein-Westfalen und des Westdeutschen Rundfunks teil. Nach einem gemeinsamen Rundgang durch die Ausstellung diskutierten die Experten unter der Gesprächsleitung Wilhelm Wessels. Anschließend fuhren die Kritiker nach Köln, wo Rolf Wiesselmann das oben erwähnte Rundfunkgespräch über dieselben Fragen moderierte. Der Westdeutsche Rundfunk sendete dieses knapp 40minütige Gespräch in voller Länge sowie mit einer Einführung und einem Schlußwort von Carl Linfert am Freitag, den 1. März 1957, ab 22.10 Uhr in seiner Reihe "Nachtprogramm «. ${ }^{8}$ In Leverkusen wie in Köln ging es um folgende drei Fragen (D):

7 Eine Photokopie der 20seitigen Dokumentation befindet sich im Stadtarchiv Leverkusen.

8 Ein Tonband der Radiosendung ist im Archiv des WDR in Köln vorhanden, wo ich Klaudia Wilde für freundliche Unterstützung danke. Jeder Teilnehmer der Runde sprach in seiner Sprache, die fremdsprachigen Beiträge wurden anschließend für die Zuhörer auf deutsch zusammengefaßt. Die folgende Rekonstruktion der Diskussion basiert einerseits auf dem Tonband, andererseits auf der 


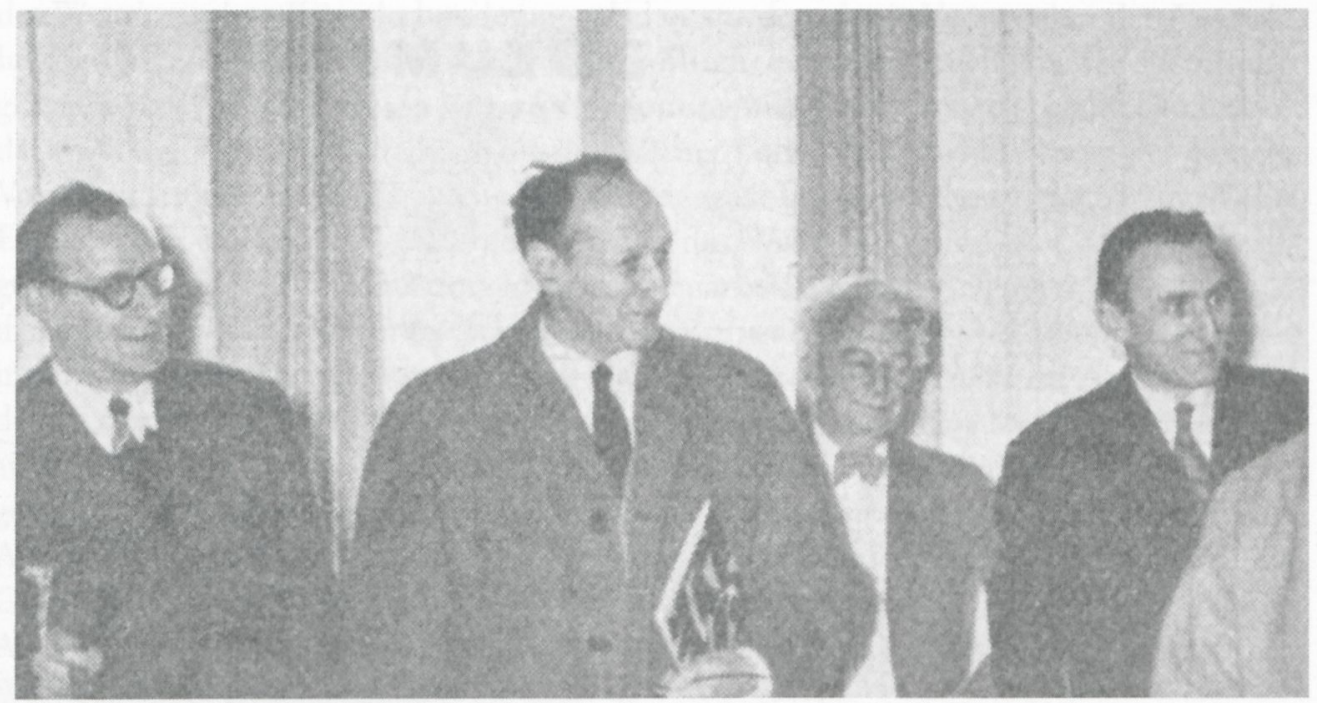

3 Ernest Goldschmidt, Pierre Janlet, Willem J. H. B. Sandberg und Michel Tapié beim »Leverkusener Gespräch«, Leverkusen, 1. Dezember 1956

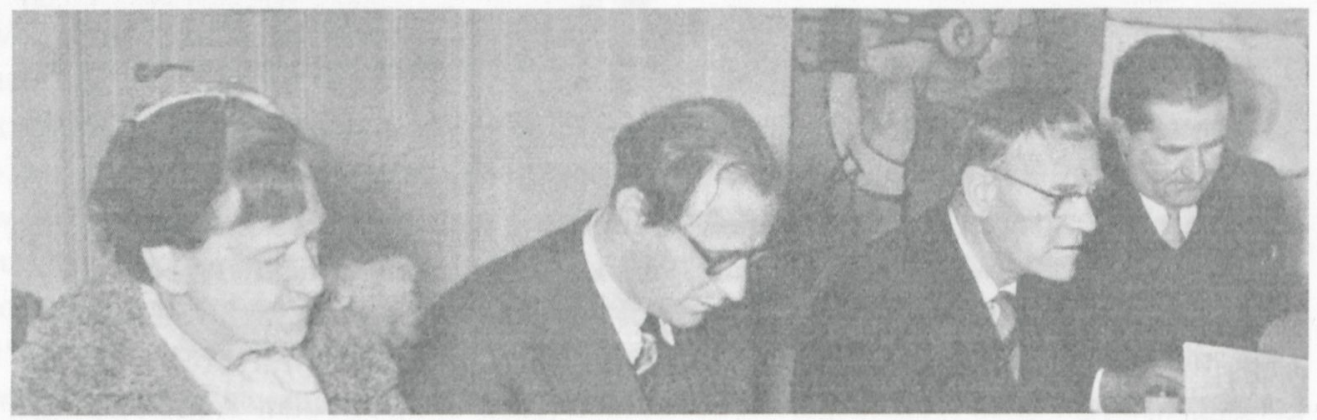

4 Herta Wescher, Ernest Goldschmidt, Georg Schmidt und Giuseppe Marchiori beim »Leverkusener Gespräch«, Leverkusen, 1. Dezember 1956

1. Ist der Durchbruch der "non-figurativen Kunst " nach 1945 in den verschiedenen Ländern als Ausdruck der »libération « zu verstehen?

2. Sind innerhalb der internationalen Bewegung der "non-figurativen Kunst " in den verschiedenen Ländern nationale Wesenszüge zu erkennen?

3. Hat die deutsche Kunst seit 1945 die Zone der Isolierung (1933-1945) überwunden?

Bei der Beantwortung der ersten Frage treten innerhalb der Runde unterschiedliche Ansichten hervor. So meint Georg Schmidt, dies sei von Land zu Land unterschiedlich

erwähnten schriftlichen Dokumentation. Zitate oder Informationen aus dem Tonband sind mit (B) kenntlich gemacht, solche aus der Dokumentation mit (D). 


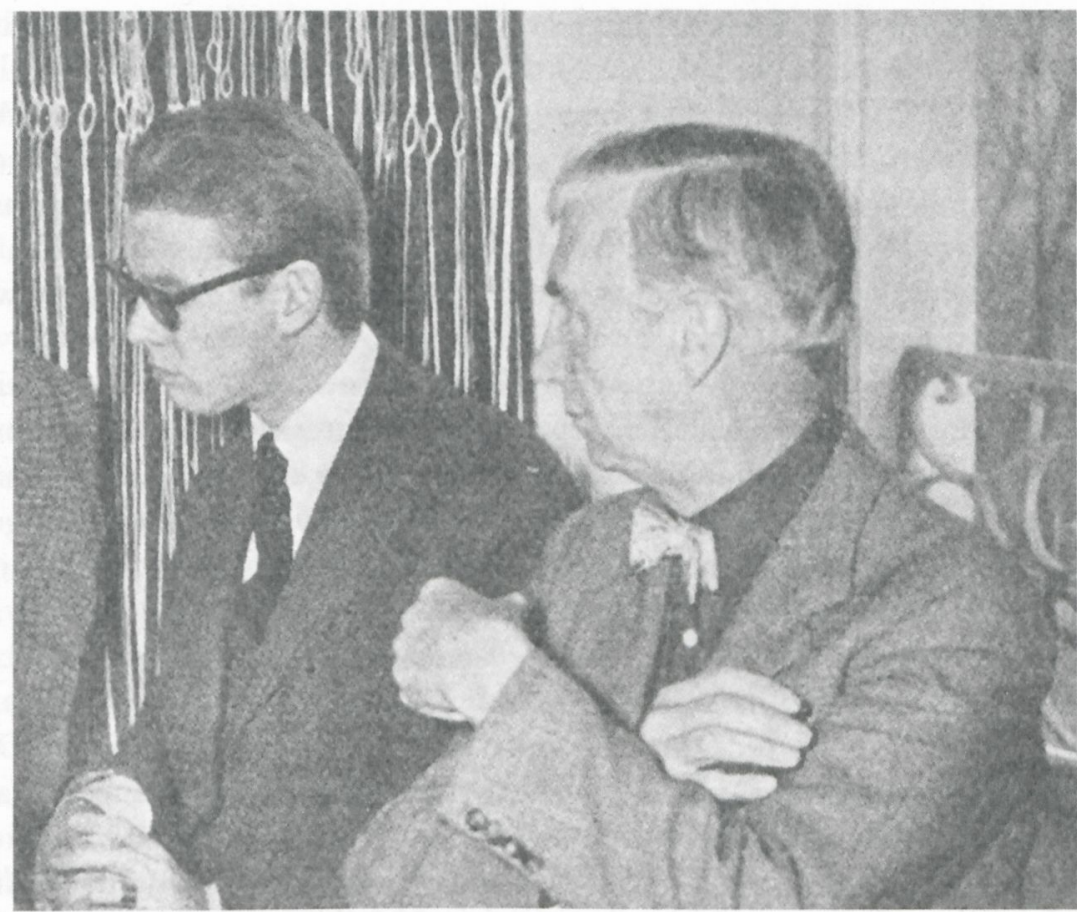

5 Rodolphe Stadler und Herbert Read beim »Leverkusener Gespräch«, Leverkusen, 1. Dezember 1956

(B), »für Deutschland und Italien« könne sie hingegen »mit einem klaren Ja beantwortet werden «(D). Zugleich warnte er davor, »den Geist der >libération< mit dem Geist der ungegenständlichen Kunst zu identifizieren und die >libération< gar als Vater der ungegenständlichen Kunst zu bezeichnen. Die >libération w war vielmehr ein Sammelbecken sämtlicher von den Diktaturen verfolgten geistigen Bewegungen«(D). Herta Wescher bemerkt zur Bedeutung der zeitgeschichtlichen Ereignisse in ihrer in der Zeitschrift "Cimaise « erschienenen Rezension, »daß die universale Bildsprache unserer Tage, die den Künstlern aller Länder gemeinsam ist, ihre Quellen in den Erschütterungen der Kriegsjahre hat, die viel zu tief, viel zu umwälzend waren, als daß sie der Kunst nicht einen grundsätzlich neuen Stempel aufprägen mußten« (D). ${ }^{9}$ Dagegen betont Giuseppe Marchiori im Hinblick auf die Situation in Italien, daß es auch unter dem Faschismus avantgardistische, abstrakt arbeitende Gruppen gegeben habe (B), und auch Ernest Goldschmidt bestreitet, daß die Abstraktion eine Folge der Befreiung gewesen sei, vielmehr habe diese neuen Schwung in eine seit Anfang des Jahrhunderts sich entwickelnde Richtung gebracht (B). Daß zum Zeitpunkt der Befreiung im Grunde schon alle Wege in der Kunst begangen waren und erst danach, mit Wols, Dubuffet und Hartung,

9 Vgl. zu Herta Wescher jüngst Martin Schieder, Im Blick des Anderen. Die deutsch-französischen Kunstbeziehungen 1945-1959, Berlin 2005 (Passagen/Passages; 12), 166-176, zu Leverkusen $173 f$. 
Künstler mit neuen Handschriften hervorgetreten seien, bemerkt Michel Tapié (B), und Will Grohmann schildert das abrupte Ende der regen Kunstentwicklung in Deutschland im Jahre 1933 (B). In diesem Sinne argumentiert auch Herbert Read. Abstraktion sei »eine unvermeidbare Tendenz innerhalb der Gegenwartskunst«, der die "politische Befreiung « eine freiere Ausdrucksart ermöglicht habe, »sei es in Deutschland oder anderswo. Beziehungen zwischen abstrakter Kunst und soziologischen Faktoren sollten auf einer tiefer liegenden Basis gesucht werden, die in der gesamten westlichen Welt anzutreffen sind (D).

In bezug auf Frage zwei waren die Experten überwiegend der Meinung, daß die (ungegenständliche) Kunst der Gegenwart eher international denn national oder regional sei, was das Vorhandensein typischer nationaler Traditionen, Merkmale, Färbungen und Nuancen jedoch durchaus einschließe. Georg Schmidt: »Die ungegenständliche Kunst ist das Produkt von international wirksamen geistigen Bedingungen der westlichen Kultur des 20. Jahrhunderts. Die ungegenständliche Kunst ist in ihren geistigen Fundamenten nicht internationaler als irgendein Stil der Vergangenheit [...]. Auch wenn kein Stil absichtlich national gefärbt sein kann [...], so ist es doch unvermeidlich, daß jeder Stil in jedem Lande ungewollt eine nationale Färbung annimmt (D). Pierre Janlet vertritt die Auffassung, die Künstler seien nicht in nationalen Eigenschaften befangen, allenfalls mit der Ausnahme lyrischer Richtungen (B). Die »neue Malerei« gestatte »eine breitere Basis von Experimenten« in aller Welt, was zu internationalem Austausch führe: »Diese gemeinsamen Erfahrungen ziehen Verallgemeinerungen in ihrer Zielsetzung nach sich - zum Nachteil nationaler Gesichtspunkte«(D). Ebenso unterstreicht Willem Sandberg den internationalen Charakter der bildenden Kunst im 20. Jahrhundert, im Unterschied zur Literatur (B). Daß im Bereich der informellen Malerei gleichwohl die jeweiligen Traditionen im Land eine Rolle spielen und zu unterschiedlichen Nuancierungen führen, betont Herta Wescher (B), und auch Georg Schmidt akzentuiert die Bedeutung der nationalen Traditionen, gibt jedoch zu bedenken, daß sich die daraus resultierenden Nuancen erst in größerem historischen Abstand zu erkennen geben (B, D). Giuseppe Marchiori ist ebenfalls der Meinung, die abstrakt arbeitenden Künstler würden »ihre nationale Diktion, ihre typischen Merkmale, ihr entsprechendes Gefühl « (B) behalten. Einigkeit besteht weitgehend darin, daß die Kunst bereits in früheren Epochen einen internationalen Charakter gehabt habe. Die Internationalisierung, so Herbert Read, werde in der Moderne durch die Verbreitung von Reproduktionen und den Austausch von Rezensionen verstärkt, was unausweichlich zu einer Beeinflussung der Künstler führe (B). Dem pflicht Ernest Goldschmidt bei, weist indes zugleich darauf hin, daß diese Form des internationalen Austauschs in der Kunstgeschichte kein neues Phänomen sei (B).

Einig ist man sich auch hinsichtlich der dritten Frage, ob die deutsche Kunst elf Jahre nach Kriegsende die Phase der Isolierung während der NS-Zeit überwunden habe. Dies könne laut Georg Schmidt »kurz und bündig mit Ja beantwortet werden« (D), womit aber auch gewisse Gefahren verbunden seien: "Sogar soweit ist Deutschland im internationalen Gespräch [der ungegenständlichen Kunst, C. Z.], daß es auch alle Gefahren 
dieser nun internationalen Mode [...] mitmacht und daß Deutschland vor den genau gleichen Problemen steht wie wir alle, nämlich, es handelt sich nicht um eine Prinzipfrage, ob abstrakt oder nicht abstrakt, sondern um eine reine Qualitätsfrage: Wer hat in dieser Sprache etwas wirklich Neues zu sagen? (B). Giuseppe Marchiori: »Die deutsche Kunst nimmt wieder aktiv teil an dem Erneuerungsprozeß, der sich in allen europäischen Ländern außerhalb des Eisernen Vorhanges - wohl aber in Polen und Jugoslawien - auswirkt « (D). Herbert Read: »Deutschland [hat sich] wieder mit der internationalen Entwicklung der Kunst vereinigt«(D). Pierre Janlet: »Deutsche Künstler nehmen Teil am Konzert der Nationen « (B). Willem Sandberg: »ich glaube, daß wir behaupten können, daß seit anfang unseres jahrhunderts wieder eine europäische kunst im entstehen ist und daß auch viele deutsche künstler an dieser entwicklung teilnahmen und -nehmen. die ausstellung in morsbroich ist davon eine bestätigung " (D). ${ }^{10}$

An dieser Stelle lenkt Rolf Wiesselmann im Rundfunkgespräch die Frage auf die Verkaufschancen deutscher Künstler im Ausland. Rodolphe Stadler sagt hierzu, er habe noch keine Erfahrungen mit deutschen Künstlern, habe von diesen bislang nur Wols ausgestellt, und der lasse sich heute überall verkaufen. ${ }^{11}$ Während Will Grohmann - der in der Dokumentation nicht mit einem Statement vertreten ist und sich, wie auch Michel Tapié, im Rundfunkgespräch merklich zurückhält - der Auffassung ist, daß sich deutsche Künstler im Ausland schlecht verkaufen lassen (mit Ausnahme Amerikas wegen der zahlreichen Emigranten), argumentiert Wilhelm Wessel, junge deutsche Künstler verkauften auswärtig sehr wohl recht gut und zwar besser als ältere Künstler (alle B).

Auf die Frage nach der Isolation der deutschen Kunst zurückkommend, ist Ernest Goldschmidt der Meinung, dies könnten letztlich nur die Deutschen selbst beantworten: "Solange Deutschland sich isoliert fühlt, bleibt es isoliert. " Georg Schmidt findet diesen Gedanken »hervorragend «: »Es kommt auch auf Deutschland an, daß es sich nicht isoliert fühlt« (beide B). Mit diesem Satz endet das Rundfunkgespräch.

\section{Das »Leverkusener Gespräch « im Kontext seiner Zeit}

Wie läßt sich das »Leverkusener Gespräch« im Kontext der Debatten und Diskurse der 50 er Jahre inhaltlich bewerten und historisch verorten? Fest steht, daß es eine vergleichbare Diskussion prominenter Museumsleiter und Kunstkritiker aus mehreren europäischen Ländern über die Situation der Kunst in West-Deutschland in dieser Zeit wohl nicht gegeben hat. Das »Leverkusener Gespräch« ist im Zusammenhang mit den kunstpolitischen Aktivitäten Wilhelm Wessels zu sehen, der sich in seiner Funktion als

10 Kleinschreibung im Original.

11 Später zeigte Stadler in seiner Pariser Galerie unter anderem Wilhelm Wessel und Emil Schumacher. Vgl. hierzu Zuschlag 2006 (wie Anm. 1). Daß Stadler Wols ausgestellt habe, läßt sich allerdings anhand der Galeriechronik nicht nachweisen. Vgl. Galerie Stadler, 30 ans de rencontres, de recherches, de partis pris 1955-1985, Paris 1985 (Privatdruck). 
Vorsitzender des Westdeutschen Künstlerbundes seit Jahren für die Anerkennung der deutschen Kunst im Ausland und umgekehrt für die Präsentation ausländischer Künstler im Inland einsetzte. ${ }^{12}$ Wessel zeichnete sowohl für die Ausstellung als auch für die Zusammensetzung der Expertenrunde und die ihr vorgegebenen Themen verantwortlich. Er verfügte über die entsprechenden Kontakte und Sprachkenntnisse, um ein solches Treffen zu organisieren. Es lag ihm, der ja selbst Künstler war, daran, durch die ausländischen Gutachten die deutsche Kunst in der internationalen Wahrnehmung aufzuwerten und damit nicht zuletzt ihre Marktchancen zu verbessern.

Dabei erinnert die Konstellation, Expertenrunde mit Rundfunkübertragung in Verbindung mit einer Ausstellung, an Darmstadt 1950. Hier hatte, fünf Jahre nach Kriegsende und ein Jahr nach Gründung der beiden deutschen Staaten, ebenfalls im Rahmen einer Ausstellung (das menschenbild in unserer zeit) ein Expertengespräch stattgefunden, das als erstes "Darmstädter Gespräch" Geschichte schrieb. Neben Künstlern, Kunsthistorikern und Kritikern nahmen auch Wissenschaftler anderer Disziplinen teil, so etwa aus Theologie, Soziologie, Medizin, Physik und Philosophie. Im "Darmstädter Gespräch« kulminierte die Auseinandersetzung um gegenständliche und ungegenständliche Kunst und um das Menschenbild in der Moderne zum ersten Mal. Hauptkontrahenten damals waren Willi Baumeister und Hans Sedlmayr, die beide nach dem Krieg mit programmatischen kunsttheoretischen Schriften an die Öffentlichkeit getreten waren. ${ }^{13}$ Der Hessische Rundfunk schnitt das Gespräch mit und sendete an zwei Terminen Ausschnitte davon (am 17. Juli und am 2. August 1950).

Ende 1956, ein Jahr nach der ersten documenta, war die historische und kunsthistorische Situation eine andere. In den Pariser Verträgen vom 5. Mai 1955 hatten die Westmächte die Bundesrepublik Deutschland in die Unabhängigkeit entlassen und ihr die Aufstellung eigener Streitkräfte im Rahmen der Westeuropäischen Union und der NATO ermöglicht. Bundeskanzler Konrad Adenauer setzte seine Politik der Westintegration fort. Außenpolitisch wuchs die Rolle der BRD in der internationalen Staatengemeinschaft, während die Wirtschaft im Lande prosperierte ("Wirtschaftswunder "). Es waren die Jahre des Kalten Krieges mit dem Ostblock, in dem auch die Kunst instrumentalisiert wurde. Die abstrakte Kunst galt als Kunst der Freiheit und als Gegenentwurf zu dem in der DDR proklamierten Sozialistischen Realismus sowjetischer Prägung. Auch in der Kunst hatte sich viel getan. Daß im Ringen um gegenständliche Kunst und ungegenständliche Kunst letztere mittlerweile international den Sieg davongetragen hatte, bezweifelte in Leverkusen niemand mehr. In Deutschland hatte sich das Informel, beginnend mit der Quadriga-Ausstellung im Dezember 1952 in Frankfurt am Main, an verschiedenen Orten manifestiert und im Inland und Ausland erste Achtungserfolge erzielt. Es sollte 1959 auf der II. documenta seinen großen Auftritt haben. In deren

12 Vgl. hierzu Zuschlag 2006 (wie Anm. 1).

13 Vgl. Willi Baumeister, Das Unbekannte in der Kunst, Stuttgart 1947. Hans Sedlmayr, Verlust der Mitte. Die bildende Kunst des 19. und 20. Jahrhunderts als Symptom und Symbol der Zeit, Salzburg 1948. 
Katalog schrieb Werner Haftmann den berühmt gewordenen Satz: »Die Kunst ist abstrakt geworden. $\aleph^{14}$

Während es in Darmstadt ein Gespräch unter Deutschen war (mit Ausnahme des Schweizers Johannes Itten waren alle Vortragenden Deutsche, genauer: Westdeutsche), die ihren Blick auf die Entwicklung der modernen Kunst richteten, befanden in Leverkusen ausländische Kritiker über die aktuelle Kunstsituation in Deutschland. Und genau hierin liegt der zentrale Aspekt. Denn Ziel der Leverkusener Ausstellung und des "Leverkusener Gesprächs« war es zu demonstrieren, daß die deutsche Kunst wieder Anschluß an die internationale Avantgarde gefunden hatte, und dies sollte durch die Sicht von außen bestätigt werden. Während es in Darmstadt in teils heftigen, emotional geführten Diskussionen um die Alternative gegenständliche oder ungegenständliche Kunst ging und man darin regelrecht zwei gegensätzliche Ideologien sah, herrschte in Leverkusen weitgehend Harmonie. Hier hatten sich, handverlesen von Wilhelm Wessel, Befürworter und Förderer der nichtfigurativen Kunst zusammengefunden, deren Anschauungen wohl in einzelnen Punkten differierten, nicht aber grundsätzlich voneinander abwichen.

Warum fiel das "Leverkusener Gespräch «, trotz der großen Beachtung, die es anfangs in den Medien erfuhr, im Gegensatz zum »Darmstädter Gespräch « so schnell dem Vergessen anheim? Eine Rolle spielt sicherlich, daß die Dokumentationsbroschüre des Westdeutschen Künstlerbundes nicht eine solche Verbreitung erfuhr wie die Buchpublikation von Hans Gerhard Evers zum »Darmstädter Gespräch «. ${ }^{15}$ Der wesentliche Grund scheint mir aber darin zu liegen, daß die in Leverkusen und Köln verhandelten Fragen nur für eine ganz kurze Zeitspanne Mitte der 50er Jahre brisant waren - und dann ganz schnell wieder obsolet. Schon 1959 war vieles von dem, was in Leverkusen und Köln noch Stoff für Diskussionen bot, völlig unstrittig. Dies belegt etwa die $I I$. documenta, wo die junge deutsche Kunst ganz selbstverständlich als Teil der internationalen Avantgarden gezeigt wurde. Vom Gefühl der Isolation keine Spur mehr. Andere Fragen rückten nun in den Fokus, etwa die, ob die moderne Kunst "gemanagt « werde. Unter dieser Fragestellung veranstaltete der Verlag der Zeitschrift »Das Kunstwerk 1959 die Baden-Badener Kunstgespräche, an denen unter anderem HAP Grieshaber teilnahm. ${ }^{16}$

14 Malerei nach 1945. Einführung von Werner Haftmann, in: II. documenta '59. Kunst nach 1945, Ausstellungskatalog Kassel, Band 1: Malerei, Köln [1959], 11-19, hier 17.

15 Vgl. Hans Gerhard Evers (Hrsg.), Das Menschenbild in unserer Zeit, Darmstadt [1950] (Darmstädter Gespräch; 1). Die Literatur zur Kontroverse um die Abstraktion in den 1950er Jahren ist mittlerweile sehr umfangreich. Vgl. etwa Karl-Ludwig Hofmann, "Als ob es so etwas wie eine Kunst gäbe! «. Anmerkungen zur Kontroverse um die abstrakte Kunst in den 50er Jahren, in: Christoph Zuschlag/Hans Gercke/Annette Frese (Hrsg.), Brennpunkt Informel. Quellen Strömungen - Reaktionen, Ausstellungskatalog Heidelberg 1998/99, Köln 1998, S. 158-165.

Vgl. Wird die moderne Kunst gemanagt? Baden-Baden/Krefeld 1959. 
Das »Leverkusener Gespräch « zeigt, daß die Aufarbeitung der kunstgeschichtlichen Situation der 1950er Jahre teilweise noch erhebliche Lücken aufweist. Dabei lohnt sich allemal auch ein Blick in die belletristische Literatur der Zeit. So findet sich in Wolfgang Koeppens 1951 erschienenem Roman »Tauben im Gras « eine Passage, in welcher der Ich-Erzähler Naturwissenschaften und ungegenständliche Kunst zusammenbringt und mit all dem nichts anzufangen weiß: »[...] die Quanten und das Leben, die Physiker quälen sich jetzt mit der Biologie, ich kann ihre Bücher nicht lesen, zu viel Mathematik Formelkram abstraktes Wissen Gehirnakrobatik, ein Leib ist kein Leib mehr, Auflösung der Gegenständlichkeit in den Bildern der neuen Maler, das sagt mir nichts, ich bin Doktor, vielleicht zu ungebildet, habe auch keine Zeit [...]. $\ll^{17}$

17 Wolfgang Koeppen, Tauben im Gras, Stuttgart/Hamburg 1951, 74. 